\title{
Design and movement trail analysis of a life testing machine for self-lubricating rod end spherical plain bearing of a helicopter
}

\author{
Zhanshan WANG ${ }^{*, * *}$, Yulin YANG ${ }^{* * * *}$, Xiping LIU ${ }^{*, * *}$ and Shijun HUANG*** \\ ${ }^{*}$ College of Mechanical Engineering, Yanshan University, Qinhuangdao, Hebei 066004, P. R. China; \\ E-mail:yang_yulin123@163.com \\ **Aviation Key Laboratory of Science and Technology on Generic Technology of Self-Lubricating Spherical Plain Bearing, Yanshan \\ University, Qinhuangdao, Hebei 066004, P. R. China
}

Received 9 May 2016

\begin{abstract}
In this paper, a new type of mechanism in a life testing machine for self-lubricating rod end spherical plain bearing of a helicopter is proposed based on the main rotor structure and motion process of a single rotor helicopter with hinged blades. The forward displacement of the mechanism is analyzed by using coordinate transformation and a function relationship for rotation variation between inner and outer ring of testing bearing rotating around the radial and axial is gained; a novel life testing machine is accomplished based on the mechanism, and a $3 \mathrm{D}$ model is built and the function curves are obtained with MATLAB. The function relationship is proved to be validity by contrasting with the motion simulation results. This life testing machine can simulate pitching and flapping motions of self-lubricating rod end spherical plain bearing of helicopter on the ground, and the research provides a theory basis for accurately applying the load on the testing bearing and boundary conditions for optimizing the mechanism structure.
\end{abstract}

Key words : Self-lubricating rod end spherical plain bearing, Life testing machine, Pitch link, Movement trail analysis, Motion simulation

\section{Introduction}

The self-lubricating rod end spherical plain bearing is widely used in main rotor system of the single rotor helicopter with hinged blades, it influences the pitching and flapping motions of a helicopter rotor. The performance of rod end spherical plain bearing affects the helicopter's control ability, and the life of rod end spherical plain bearing affects the helicopter's maintenance period and service life (Gao, et al.,2006). The flight fault caused by the failure of spherical plain bearing has occurred many times (McFalls M, et al., 2004a, McFalls M and Jackson A, et al.,2004b) Therefore it is necessary to fully test the performance and evaluate the life of the rod end spherical plain bearing.

Recently, the research about spherical plain bearing is conducted by developing testing equipment to simulate the actual working and loading condition and evaluate performance and service life of rod end spherical plain bearing. The alternating loading spherical plain bearing life testing machine designed by Changchun research institute for mechanical science CO.LTD (CIMACH) can be used to test alternating loading of joint bearing (Fan, et al., 2011) .The modular joint bearing life testing machine designed by Yanshan University (Zhou, 2012), the oscillatory wear performance testing machine for spherical plain bearing made by NTN Toyo bearing CO. (Shimizu T, et al., 1982) and the variable load spherical plain bearing life tester developed by Royal aircraft establishment (Lancaster J K, 1983) can be used to apply low frequency oscillation and heavy loading on the testing bearing. These testing machines can only simulate one-dimensional loading, however the self-lubricating rod end spherical plain bearing need to bear multidimensional loading. Thus, these testing machines cannot meet the requirement of complex motion simulation. The spherical plain bearing tester of Swash plate designed by Yanshan University (Wei, et al., 2008) and spherical plain bearing tester of Swash plate developed by Boeing helicopter company(Jonathan A. Keller and Paul Grabill, 2005) can achieve the simulation of helicopter flight motion with multidimensional loading. The two kinds of tester can only be used to test the spherical plain bearings of swash plate, so it lacks of testing machines in view of self-lubricating rod end spherical plain bearing. 
In this paper, a new type of the life testing machine based on the 3-(R)RSS-(R)RRS-PS parallel mechanism for self-lubricating rod end spherical plain bearing of the helicopter is put forward by referencing to the main rotor structure and motion process of the single rotor helicopter with hinged blades and ignoring tiny changes of the blade angle. The actual movement of testing bearing under pitching and flapping motions can be simulated and analyzed via the machine on the ground. This paper supplies a theory basis for accurately applying the loading on the testing bearing and boundary conditions for optimizing the mechanism structure.

\section{Design for mechanism}

The pitch link of main rotor system of the single rotor helicopter with hinged blades connects the swash plate to pitch swing arm so as to transfer motion and control the paddle. The self-lubricating rod end spherical plain bearings installed on both ends of pitch link offer the degree of spatial freedom to the movement of the pitch link.

Self-lubricating rod end spherical plain bearing consists of inner ring of spherical plain bearing outer ring with screw of spherical plain bearing and self-lubricating fabric liner. The self-lubricating fabric liner is weaved by PTFE and Kevlar, it has excellently self-lubricating property. The self-lubricating rod end spherical plain bearings have three DOF and they are maintenance-free. They are applied in abominable working condition.

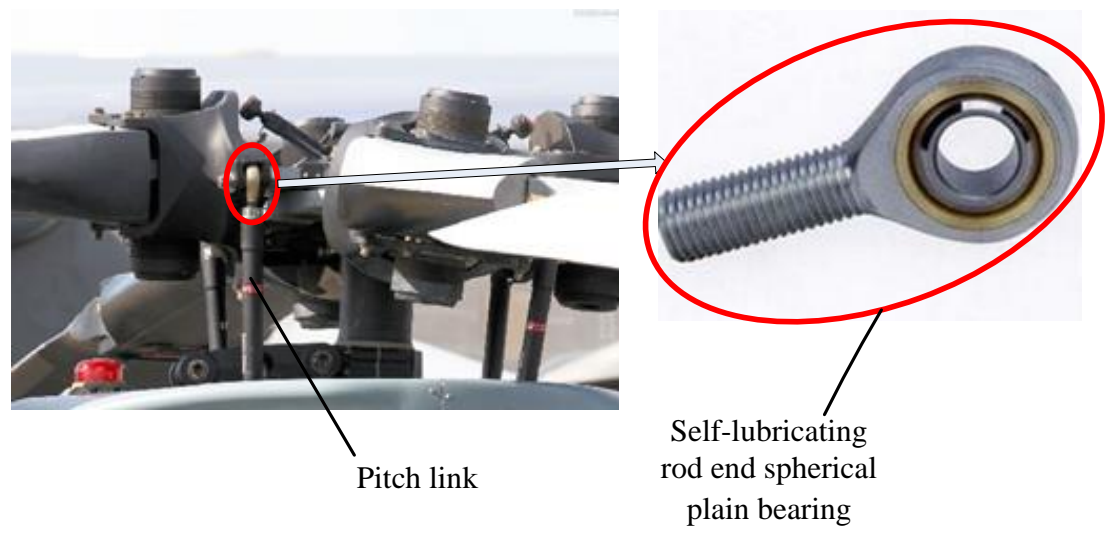

Figure 1. Self-lubricating rod end spherical plain bearing of a helicopter

The working speed of spindle of helicopter is 200r/m 400r/m (Qin, et al., 2007, Lin, et al., 2016), the period of motion of self-lubricating rod end spherical plain bearing is agreed with spindle. Because of sustaining the pitching and flapping motions, the movement of self-lubricating rod end spherical plain bearing is complex and varied. To accurately simulate the motions and evaluate the lifetime of testing bearings on the ground, the life testing machine must meet the following requirements:

1) The spindle of life testing machine can rotate at $200 \mathrm{r} / \mathrm{m} \sim 400 \mathrm{r} / \mathrm{m}$ to simulate the motion of paddles;

2) The life testing machine can simulate every oscillating motions of splash plate, and implement the pitching motions and the total distance movement;

3) The life testing machine can implement the flapping motions.

\subsection{Configuration design}

Professor Guo Xijuan (Guo, et al., 2013, Wang, et al., 2014, Zhou, et al., 2014) studied the main rotor structure of the single rotor helicopter with hinged blades, and put forward an equivalent mechanism. By referring to the equivalent mechanism, we design a novel mechanism of life testing machine for self-lubricating rod end spherical plain bearing of the helicopter. The schematic diagram of mechanism is shown in Figure2.

The life testing machine has upper and lower platform. The upper platform represents a helicopter blade, and the lower platform represents a swash plate. The lower platform connects to the foundation through moving pair $\mathrm{P}$ and ball friction coupling $\mathrm{S}$, and the upper platform connects to the lower platform through three identical support arms $\mathrm{D}_{i} \mathrm{E}_{i} \mathrm{~F}_{i} \mathrm{G}_{i} \mathrm{H}_{i}(i=1 \sim 3)$ and a torque arm structure $\mathrm{D}_{4} \mathrm{E}_{4} \mathrm{~F}_{4}$. This structure guarantees the upper platform and lower platform can synchronously rotate. The three identical support arms $\mathrm{D}_{i} \mathrm{E}_{i} \mathrm{~F}_{i} \mathrm{G}_{i} \mathrm{H}_{i}(i=1 \sim 3)$ represent pitch links, and the testing bearings are installed on the ends of the support arms. A CAM mechanism is arranged in each of the branched chain $\mathrm{G}_{i} \mathrm{H}_{i}(i=1 \sim 3)$, as a passive drive to apply flapping motions on the testing bearing. 


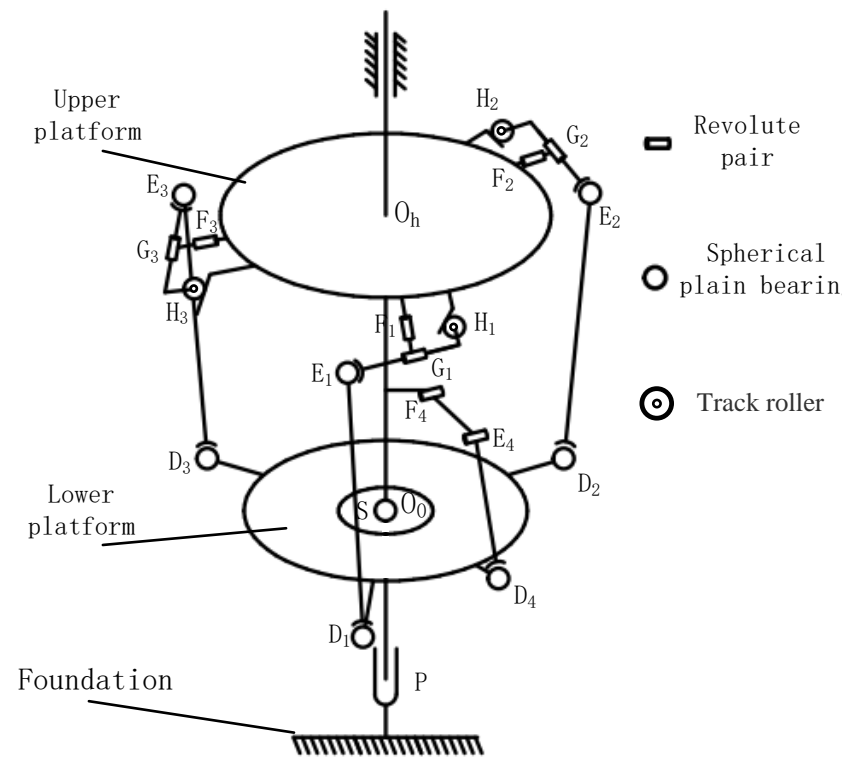

\subsection{DOF analysis}

Figure.2 Schematic diagram of life testing machine

Every support arm connects with the upper and lower platforms. The upper platform, the support arm and the lower platform compose a double closed-loop serial mechanism. Thereinto, the CAM mechanism is a passive drive to apply flapping motions on the testing bearing, so the DOF of CAM mechanism does not affect the whole DOF of the life testing machine. When the DOF of the mechanism is calculated, we can ignore the CAM mechanism and get a simple schematic diagram as shown in the Figure 3.

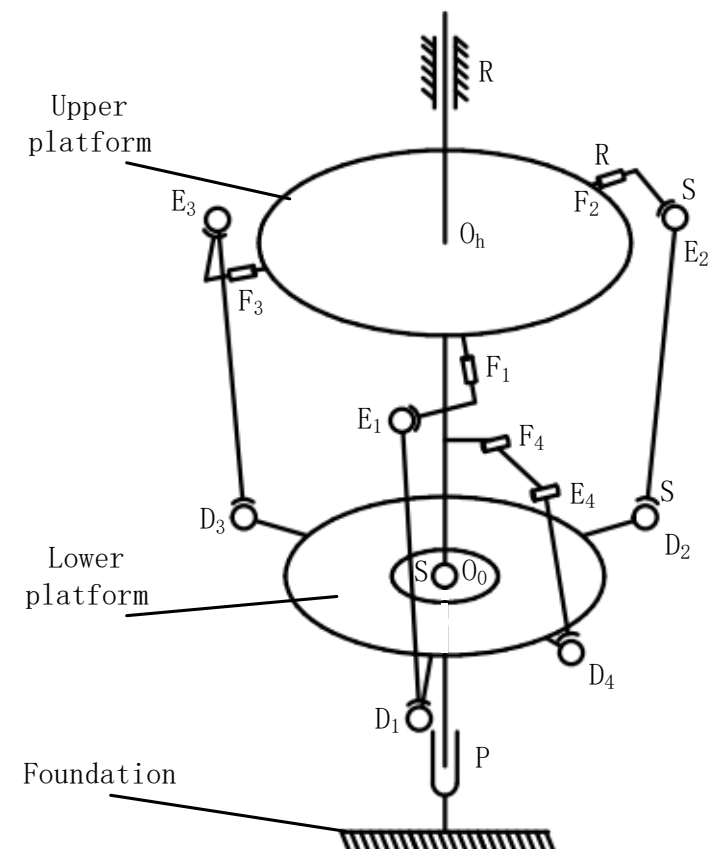

Figure. 3 Schematic diagram of life testing machine without the adjusting flapping system

The segment $\mathrm{O}_{0} \mathrm{O}_{\mathrm{h}}$ represents the spindle of mechanism in the figure 3, and the mechanism body rotates around the spindle. The rotation can be equivalent to a revolute pair $\mathrm{R}$. The spindle connects on the upper platform. The spindle and the upper /lower platform can synchronously rotate with three support arms $\mathrm{D}_{i} \mathrm{E}_{i} \mathrm{~F}_{i} \mathrm{G}_{i} \mathrm{H}_{i}(i=1 \sim 3)$ and the torque arm $\mathrm{D}_{4} \mathrm{E}_{4} \mathrm{~F}_{4}$. The rotating components and three support arms are equivalent to 3-(R)RSS, and the spindle is equivalent to (R). Torque arm $\mathrm{D}_{4} \mathrm{E}_{4} \mathrm{~F}_{4}$ is equivalent to RRS, and it is used to connect the spindle. Finally the mechanism structure is equivalent to (R) RRS. The PS institution is designed to realize the posture adjustment of the lower platform. So the total structure can be simplified as 3-(R)RSS-(R)RSS-PS. 
According to the Kutzbach-Grüber criterion $\mathrm{M}=\mathrm{d}(\mathrm{n}-\mathrm{g}-1)+\sum_{i=1}^{g} f_{i}+\gamma-\xi$

Where $M$ represents the mobility of mechanism, $d$ represents the order of mechanism, $d=6-\lambda, \lambda$ represents the number of independent common constraints, $\mathrm{n}$ represents the number of links, $\mathrm{g}$ represents the number of joints, $\mathrm{f}_{i}$ represents the freedom of $i_{\text {th }}$ joint. $\gamma$ represents the number of redundant constraint, $\xi$ represents the number of local DOF.

In this life testing machine, $\mathrm{n}=12$ and $\mathrm{g}=15$. Every support arm has an isolated degree of freedom between $\mathrm{S}-\mathrm{S}$, and the (R) is extra degree of freedom in the (R)RSS. So the DOF of the life testing machine can be calculated according to the Kutzbach-Grüber criterion.

$M=6 \times(12-15-1)+3 \times(1+1+3+3)+(1+1+1+3)+(1+3)-3-3=4$

Through the analysis, the DOF of the life testing machine is 4 . The swash plate can move along and rotate around principal axis, and rotate around the two axes in the horizontal plane.

\subsection{Forward displacement Analysis of the mechanism}

The DOF of life testing machine is set as input, and $\phi$ represents the spindle rotation, $Z_{c}$ represents the movement along the vertical direction of the swash plate driven by the boosting system, and the Euler angles are used to describe posture of the rotating ring of the swash plate. The angle of the link $\mathrm{E}_{i} \mathrm{~F}_{i}(i=1 \sim 3)$ in every support arm is received via the space transform, when it rotates around the point $\mathrm{F}_{i}$.

In the initial state, a fixed coordinate system $\boldsymbol{O}_{\boldsymbol{o}}-\boldsymbol{X}_{\boldsymbol{o}} \boldsymbol{Y}_{\boldsymbol{o}} \boldsymbol{Z}_{\boldsymbol{o}}$ is built on the lower platform. The axis $\boldsymbol{X}_{\mathbf{0}}$ passes through the center of spherical hinge $\boldsymbol{D}_{\mathbf{1}}$, and the axis $\boldsymbol{Z}_{\mathbf{0}}$ is along the spindle and the vertical upward is positive, the direction of axial $\boldsymbol{Y}_{\mathbf{0}}$ is determined by the right-hand rule. The point $\mathrm{O}_{\mathrm{h}}$ is set as the origin of moving coordinate named $\boldsymbol{O}_{\boldsymbol{h}}-\boldsymbol{X}_{\boldsymbol{h}} \boldsymbol{Y}_{\boldsymbol{h}} \boldsymbol{Z}_{\boldsymbol{h}}$, and the upper platform is set as the plane $\boldsymbol{X}_{\boldsymbol{h}} \boldsymbol{Y}_{\boldsymbol{h}}$, the axis $\boldsymbol{Z}_{\boldsymbol{h}}$ is along the spindle and the vertical upward is positive

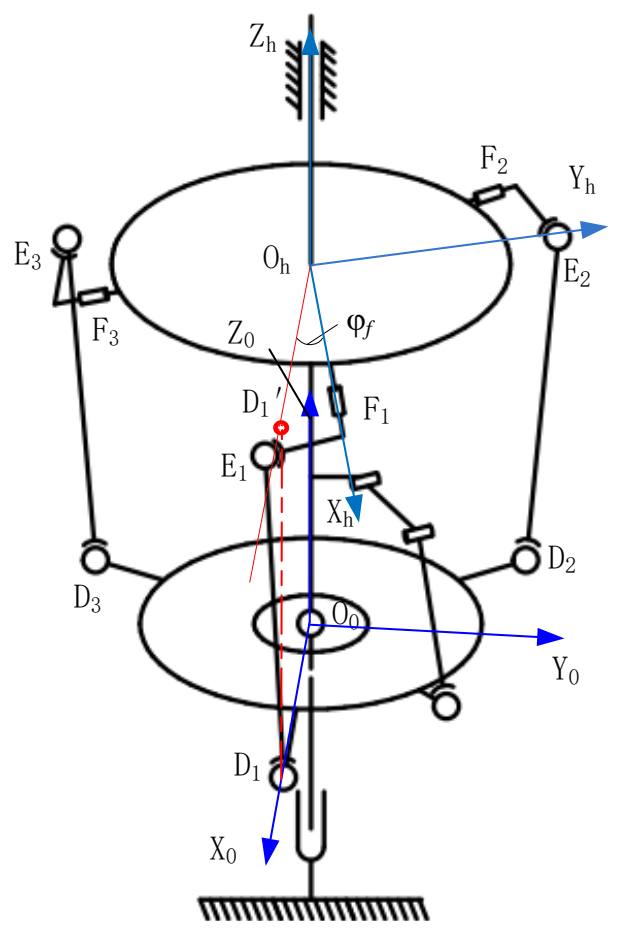

Figure.4 D-H coordinate system

The fixed coordinate system $\boldsymbol{O}_{\mathbf{0}}-\boldsymbol{X}_{\mathbf{0}} \boldsymbol{Y}_{\mathbf{0}} \boldsymbol{Z}_{\mathbf{0}}$ transfers to the moving coordinate system $\boldsymbol{O}_{\boldsymbol{r}}-\boldsymbol{X}_{\boldsymbol{r}} \boldsymbol{Y}_{\boldsymbol{r}} \boldsymbol{Z}_{\boldsymbol{r}}$ with the lower platform moving. The fixed coordinate system $\boldsymbol{O}_{\mathbf{0}}-\boldsymbol{X}_{\mathbf{0}} \boldsymbol{Y}_{\mathbf{0}} \boldsymbol{Z}_{\mathbf{0}}$ moves $\boldsymbol{Z}_{\boldsymbol{c}}$ along the axis of ordinates, and the fixed coordinate system $\boldsymbol{O}_{\mathbf{0}}-\boldsymbol{X}_{\mathbf{0}} \boldsymbol{Y}_{\mathbf{0}} \boldsymbol{Z}_{\mathbf{0}}$ transfers to the coordinate system $\boldsymbol{O}_{\mathbf{0}}^{\prime}-\boldsymbol{X}_{\mathbf{0}}^{\prime} \boldsymbol{Y}_{\mathbf{0}}^{\prime} \boldsymbol{Z}_{\mathbf{0}}^{\prime}$; then $\boldsymbol{O}_{\mathbf{0}}^{\prime}-\boldsymbol{X}_{\mathbf{0}}^{\prime} \boldsymbol{Y}_{\mathbf{0}}^{\prime} \boldsymbol{Z}_{\mathbf{0}}^{\prime}$ rotates $\psi$ around the axis $\boldsymbol{Z}_{\mathbf{0}}^{\prime}$, and the coordinate system $\boldsymbol{O}_{\mathbf{0}}^{\prime}-\boldsymbol{X}_{\mathbf{0}}^{\prime} \boldsymbol{Y}_{\mathbf{0}}^{\prime} \boldsymbol{Z}_{\mathbf{0}}^{\prime}$ transfers to the coordinate system $\boldsymbol{O}_{\mathbf{1}}-\boldsymbol{X}_{\mathbf{1}} \boldsymbol{Y}_{\mathbf{1}} \boldsymbol{Z}_{\mathbf{1}}$; then $\boldsymbol{O}_{1}-\boldsymbol{X}_{\mathbf{1}} \boldsymbol{Y}_{\mathbf{1}} \boldsymbol{Z}_{\mathbf{1}}$ rotates $\varphi$ around the axis $\boldsymbol{X}_{\mathbf{1}}$, and the coordinate system $\boldsymbol{O}_{\mathbf{1}}-\boldsymbol{X}_{\mathbf{1}} \boldsymbol{Y}_{\mathbf{1}} \boldsymbol{Z}_{\mathbf{1}}$ transfers to the coordinate system $\boldsymbol{O}_{2}-X_{2} \boldsymbol{Y}_{2} Z_{2}$; at last $\boldsymbol{O}_{2}-X_{2} \boldsymbol{Y}_{2} \boldsymbol{Z}_{2}$ rotates $\psi$ around the axis $Z_{2}$, and the coordinate system 
$\boldsymbol{O}_{\mathbf{2}}-\boldsymbol{X}_{\mathbf{2}} \boldsymbol{Y}_{\mathbf{2}} \boldsymbol{Z}_{\mathbf{2}}$ transfers to the moving coordinate system $\boldsymbol{O}_{\boldsymbol{r}}-\boldsymbol{X}_{\boldsymbol{r}} \boldsymbol{Y}_{\boldsymbol{r}} \boldsymbol{Z}_{\boldsymbol{r}}$. The Euler angle $\mathbf{Z}-\mathbf{X}-\mathbf{Z}$ is used to describe the space position of the lower platform.

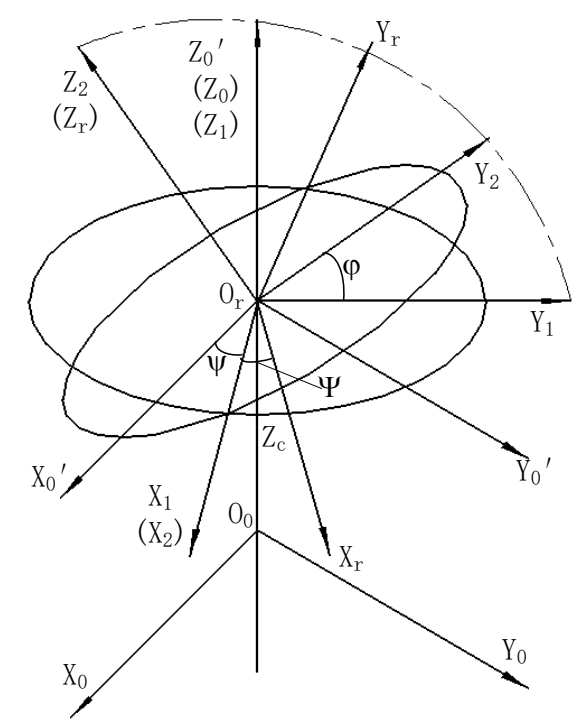

Figure.5 The transformation of Euler angle

Because the torque arm connects the spindle to swash plate, it guarantees the points of intersection between vertical plane through the spindle axis and upper/lower platform are always located in a vertical plane when they are rotating. The point $\mathrm{D}_{1}^{\prime}$ and the points $\mathrm{D}_{1}, \mathrm{O}_{\mathrm{r}}, \mathrm{O}_{\mathrm{h}}$ are coplanar, where the point $\mathrm{D}_{1}^{\prime}$ is a projection that the point $\mathrm{D}_{1}$ maps on the upper platform in the initial state. So vector $\overrightarrow{\boldsymbol{O}_{r} \boldsymbol{O}_{h}}, \overrightarrow{\boldsymbol{O}_{h} \boldsymbol{D}_{1}^{\prime}}, \overrightarrow{\boldsymbol{Z}}$ are coplanar, and the $\overrightarrow{\boldsymbol{Z}}$ is an unit vector.

$\left(\overrightarrow{O_{r} O_{h}} \times \overrightarrow{O_{h} D_{1}^{\prime}}\right) \cdot \vec{Z}=0$

At last the rotation of spindle $\phi$ is shown in equation (3) with the three Euler angle.

$\tan \phi=\frac{s \psi \cdot c \psi+\mathrm{c} \psi \cdot \mathrm{c} \varphi \cdot \mathrm{s} \psi}{c \psi \cdot c \psi-\mathrm{s} \psi \cdot \mathrm{c} \varphi \cdot \mathrm{s} \psi}$

Where $s$ represents sine, $c$ represents cosine.

According to D-H coordinate method, the coordinate of points $F_{i}, D_{i}$ located in the fixed coordinate system is:

$$
\boldsymbol{F}_{\boldsymbol{i}}=\left[\begin{array}{c}
c\left(\phi-\varphi_{f}\right) \cdot c\left(\frac{2 \pi(i-1)}{3}\right) \cdot R-s\left(\phi-\varphi_{f}\right) \cdot s\left(\frac{2 \pi(i-1)}{3}\right) \cdot R \\
s\left(\phi-\varphi_{f}\right) \cdot c\left(\frac{2 \pi(i-1)}{3}\right) \cdot R+c\left(\phi-\varphi_{f}\right) \cdot s\left(\frac{2 \pi(i-1)}{3}\right) \cdot R \\
Z_{h}
\end{array}\right]=\left[\begin{array}{l}
a \\
b \\
c
\end{array}\right]
$$

where $Z_{h}$ is the distance from the point $\mathrm{O}_{0}$ to point $\mathrm{O}_{\mathrm{h}} ; \varphi_{f}$ represents the included angle between axis $\boldsymbol{X}_{\boldsymbol{r}}$ and axis $\boldsymbol{X}_{\boldsymbol{h}}$ in the every support arm.

$$
\boldsymbol{D}_{\boldsymbol{i}}=\left[\begin{array}{l}
e \\
f \\
g
\end{array}\right]=\left[\begin{array}{c}
(c \psi \cdot c \varphi-s \psi \cdot c \varphi \cdot s \psi) \cdot r \cdot c\left(\frac{2 \pi(i-1)}{3}\right)-(s \psi \cdot c \psi+s \psi \cdot c \varphi \cdot c \psi) \cdot r \cdot s\left(\frac{2 \pi(i-1)}{3}\right) \\
(s \psi \cdot c \varphi+c \psi \cdot c \varphi \cdot s \psi) \cdot r \cdot c\left(\frac{2 \pi(i-1)}{3}\right)-(s \psi \cdot s \psi-c \psi \cdot c \varphi \cdot c \psi) \cdot r \cdot s\left(\frac{2 \pi(i-1)}{3}\right) \\
s \varphi \cdot s \psi \cdot r \cdot c\left(\frac{2 \pi(i-1)}{3}\right)+s \varphi \cdot c \psi \cdot r \cdot s\left(\frac{2 \pi(i-1)}{3}\right)+Z_{c}
\end{array}\right]
$$

The coordinate of point $\mathrm{E}_{i}$ located in the fixed coordinate system is stipulated for:

$\boldsymbol{E}_{\boldsymbol{i}}=\left[\begin{array}{c}\mathrm{X}_{i} \\ Y_{i} \\ Z_{i}\end{array}\right]=\left[\begin{array}{l}x \\ y \\ Z\end{array}\right]$

According to the mechanism design, the system of equations (7) is obtained with the geometrical relationship of three points $\mathrm{D}_{i}, \mathrm{E}_{i}, \mathrm{~F}_{i}$. 


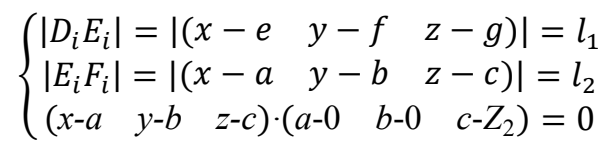

The system of equation (7) is simplified as follow:

$E+K \cdot W=2 \cdot\left(\frac{a \cdot f-b \cdot e}{a}\right) \cdot(y-b)$

Where $E=l_{2}^{2}-l_{1}^{2}+(a-e)^{2}+(b-f)^{2}+(c-g)^{2}, K=2 \cdot(c-g), W=z-c$.

Three points $\mathrm{F}_{i}, \mathrm{D}_{i}, \mathrm{E}_{i}$ form a valid triangle, so $E>0$. Because of $K=2 \cdot(c-g)>0, E K>0$. The $\mathrm{W}$ is given in equation (9).

$\mathrm{W}=\frac{-E K+\frac{2(a f-b e)}{\sqrt{a^{2}+b^{2}}} \sqrt{K^{2} l_{2}^{2}-E^{2}+\frac{4(a f-b e)^{2}}{a^{2}+b^{2}} \cdot l_{2}^{2}}}{\left[K^{2}+\frac{4(a f-b e)^{2}}{a^{2}+b^{2}}\right]}$

At last, $\alpha$ which represents rotational angle of pitch swing arm caused by pitching motions is given in equation (10).

$\alpha=\sin ^{-1}\left(\frac{W}{l_{2}}\right)$

\section{Motion function of testing bearing}

\subsection{Motion function of pitching motions}

Three pitch links connect with the upper/lower platform and the spindle, and the structure $\mathrm{O}_{\mathrm{r}}-\mathrm{D}_{\mathrm{i}}-\mathrm{E}_{\mathrm{i}}-\mathrm{F}_{\mathrm{i}}-\mathrm{O}_{\mathrm{h}}$ forms the spatial four-bar linkage, it is equivalent to RSSR mechanism. The rotation that support arms turn around the spindle can be equivalent to a rotation that $O_{r} D_{i}$ rotates around the axis $X$, which is an vertical of the plane $O_{h} O_{r} D_{i}$ and passes through the point $\mathrm{O}_{\mathrm{r}}$.

Every support arm is a double closed-loop serial mechanism. $\theta_{1}^{\prime}$ represents input variable, and $\alpha$ represents output variable. At the same time, $\alpha$ is also the input variable of the second closed-loop. So the angle of flapping motions can be calculated when value of $\alpha$ is received.

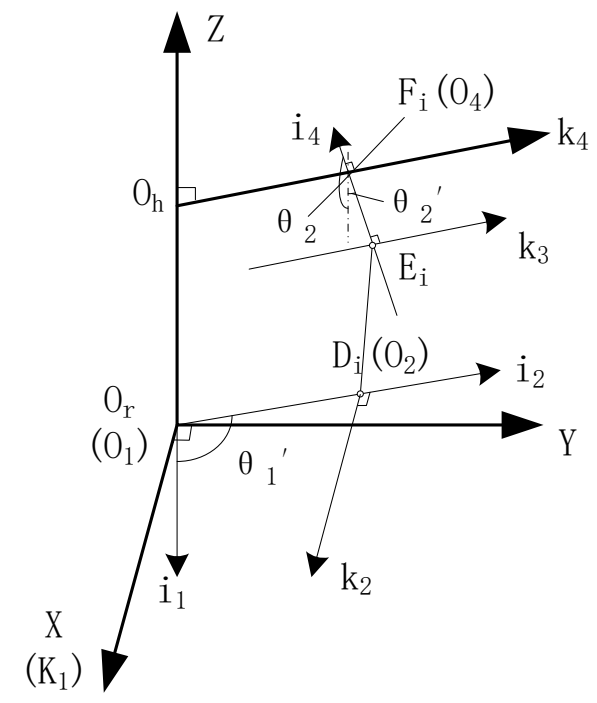

Figure.6 D-H coordinate system of the pitch link

Because the coordinates of three points $\mathrm{F}_{\mathrm{i}}, \mathrm{D}_{\mathrm{i}}, \mathrm{E}_{\mathrm{i}}$ are expressed complexity in the fixed coordinate system, a new coordinate system is built on the support arm. The point $O_{r}$ is set as the origin of coordinates $O_{1}$, axis $\boldsymbol{i}_{\mathbf{1}}$ is along the spindle vertical downward, and axis $\boldsymbol{k}_{\mathbf{1}}$ is perpendicular to the plane $\mathrm{O}_{\mathrm{h}} \mathrm{O}_{\mathrm{r}} \mathrm{D}_{\mathrm{i}}$ and passes through point $\mathrm{O}_{\mathrm{r}}$, at last a coordinate system $\boldsymbol{O}_{\mathbf{1}}-\boldsymbol{i}_{\mathbf{1}} \boldsymbol{j}_{\mathbf{1}} \boldsymbol{k}_{\mathbf{1}}$ is built. The origin of the coordinate system $\boldsymbol{O}_{\mathbf{2}}-\boldsymbol{i}_{\mathbf{2}} \boldsymbol{j}_{\mathbf{2}} \boldsymbol{k}_{\mathbf{2}}$ is point $\mathrm{D}_{i}$, and axis $\boldsymbol{i}_{\mathbf{2}}$ is along the segment $O_{r} D_{i}$, at the same time we set axis $\boldsymbol{k}_{\mathbf{2}} \| \boldsymbol{k}_{\mathbf{1}}$. The origin of the coordinate system $\boldsymbol{O}_{\mathbf{4}}-\boldsymbol{i}_{\mathbf{4}} \boldsymbol{j}_{\mathbf{4}} \boldsymbol{k}_{\mathbf{4}}$ is point $\mathrm{F}_{i}$, and axis $\boldsymbol{k}_{\mathbf{4}}$ is along the segment $O_{h} F_{i}$, and the axis $\boldsymbol{k}_{\mathbf{4}}$ is perpendicular to axis $\boldsymbol{i}_{\mathbf{1}}$ according to the mechanism, and axis $\boldsymbol{i}_{\mathbf{4}}$ is along the segment $D_{i} F_{i}$. The origin of the coordinate system $\boldsymbol{O}_{\mathbf{3}}-\boldsymbol{i}_{3} \boldsymbol{j}_{3} \boldsymbol{k}_{\mathbf{3}}$ is point $\mathrm{E}_{i}$, and 
axis $\boldsymbol{i}_{\mathbf{3}}$ is along the segment $D_{i} F_{i}$, we also set axis $\boldsymbol{k}_{\mathbf{3}} \| \boldsymbol{k}_{\mathbf{4}}$. The included angle of axis $\boldsymbol{i}_{\mathbf{1}}$ and axis $\boldsymbol{i}_{\mathbf{2}}$ is set as $\theta_{1}^{\prime}$, and $\theta_{2}$ represents the included of axis $\boldsymbol{i}_{\mathbf{4}}$ and axis $\boldsymbol{i}_{\mathbf{1}}$. Similarly $\alpha_{4}$ represents the included of axis $\boldsymbol{k}_{\mathbf{1}}$ and axis $\boldsymbol{k}_{\mathbf{4}}$.

According to the structure, $O_{1} O_{2}=\mathrm{r}, O_{h} F_{i}=R, E_{i} F_{i}=l_{2}, D_{i} E_{i}=l_{1}, O_{1} O_{h}=h_{z}$.

$\left[\boldsymbol{T}_{13}\right]$ is the transformational matrix from coordinate system 1 to coordinate system 3 :

$\left[\boldsymbol{T}_{13}\right]=\left[\begin{array}{cccc}\cos \theta_{2} & \cos \alpha_{4} \sin \theta_{2} & \sin \alpha_{4} \sin \theta_{2} & l_{2}+h_{z} \cos \theta_{2} \\ -\sin \theta_{2} & \cos \alpha_{4} \cos \theta_{2} & \sin \alpha_{4} \cos \theta_{2} & -h_{z} \sin \theta_{2} \\ 0 & -\sin \alpha_{4} & \cos \alpha_{4} & -R \\ 0 & 0 & 0 & 1\end{array}\right]$

$\left[\boldsymbol{T}_{\mathbf{2 1}}\right]$ is the transformational matrix from coordinate system 2 to coordinate system 1 :

$\left[\boldsymbol{T}_{\mathbf{2 1}}\right]=\left[\begin{array}{cccc}\cos \theta_{1}^{\prime} & \sin \theta_{1}^{\prime} & 0 & r \cos \theta_{1}^{\prime} \\ -\sin \theta_{1}^{\prime} & \cos \theta_{1}^{\prime} & 0 & r \sin \theta_{1}^{\prime} \\ 0 & 0 & 1 & 0 \\ 0 & 0 & 0 & 1\end{array}\right]$

Where $\theta_{1}^{\prime}=\frac{\pi}{2}+\theta_{1}, \theta_{2}^{\prime}=\frac{\pi}{2}+\alpha, \alpha_{4}=\frac{\pi}{2}+\varphi_{f}$.

Point $D_{i}$ is the origin of coordinate system 2, and the coordinate is $\boldsymbol{D}_{\boldsymbol{i o 2} 2}=\left[\begin{array}{lll}0 & 0 & 0\end{array}\right]$, so the coordinate of point $\mathrm{D}_{\mathrm{i}}$ in the coordinate system 1 is as follow.

$D_{i O 1}=\left[T_{21}\right] D_{i O 2}$

At last, the coordinate of point $\mathrm{D}_{i}$ in the coordinate system $\boldsymbol{O}_{\mathbf{3}}-\boldsymbol{i}_{\mathbf{3}} \boldsymbol{j}_{\mathbf{3}} \boldsymbol{k}_{\mathbf{3}}$ is shown in equation (14):

$\boldsymbol{D}_{\boldsymbol{i o 3}}=\left[\boldsymbol{T}_{\mathbf{1 3}}\right] \boldsymbol{D}_{\boldsymbol{i o 1}}\left[\boldsymbol{T}_{\mathbf{1 3}}\right]\left[\boldsymbol{T}_{\mathbf{2 1}}\right] \boldsymbol{D}_{\boldsymbol{i o 2}}=\left[\begin{array}{c}r \cos \theta_{1}^{\prime} \cos \theta_{2}+r \sin \theta_{1}^{\prime} \cos \alpha_{4} \sin \theta_{2}+l_{2}+h_{z} \cos \theta_{2} \\ -r \cos \theta_{1}^{\prime} \sin \theta_{2}+r \sin \theta_{1}^{\prime} \cos \alpha_{4} \cos \theta_{2}-h_{z} \sin \theta_{2} \\ -r \sin \theta_{1}^{\prime} \sin \alpha_{4}-R \\ 1\end{array}\right]$

In the spatial four-bar linkage, the testing bearing is installed on the position of point $\mathrm{E}_{i}$, the coordinate of point $\mathrm{E}_{i}$ in the coordinate system $\boldsymbol{O}_{3}-\boldsymbol{i}_{3} \boldsymbol{j}_{3} \boldsymbol{k}_{\mathbf{3}}$ is $\boldsymbol{E}_{\boldsymbol{i} \boldsymbol{0} \mathbf{3}}=\left[\begin{array}{lll}0 & 0 & 0\end{array}\right]$. So the coordinate of pitch link $\mathrm{D}_{i} \mathrm{E}_{i}$ in the coordinate system 3 is shown in equation (15):

$\overline{\boldsymbol{E}_{\boldsymbol{\imath}} \boldsymbol{D}_{\boldsymbol{\imath} \mathbf{3} 3}}=\left[\begin{array}{c}r \cos \theta_{1}^{\prime} \cos \theta_{2}+r \sin \theta_{1}^{\prime} \cos \alpha_{4} \sin \theta_{2}+l_{2}+h_{z} \cos \theta_{2} \\ -r \cos \theta_{1}^{\prime} \sin \theta_{2}+r \sin \theta_{1}^{\prime} \cos \alpha_{4} \cos \theta_{2}-h_{z} \sin \theta_{2} \\ -r \sin \theta_{1}^{\prime} \sin \alpha_{4}-R \\ 1\end{array}\right]=\left[\begin{array}{c}x_{D} \\ y_{D} \\ z_{D} \\ 1\end{array}\right]$

According to the coordinate of the link $E_{i} D_{i}$ in the coordinate system 3 , an angle relationship between $E_{i} D_{i}$ and axis $\boldsymbol{i}_{3}$ is obtained. Because the inner ring of testing self-lubricating rod end spherical plain bearing is fixed on the axes $\boldsymbol{i}_{3}$, and the outer ring is fixed on the pitch link $\mathrm{D}_{i} \mathrm{E}_{i}$. The plane confirmed by link $\mathrm{E}_{i} \mathrm{D}_{i}$ and axis $\boldsymbol{i}_{\mathbf{3}}$ and the plane confirmed by axis $\boldsymbol{k}_{\mathbf{3}}$ and axis $\boldsymbol{i}_{\mathbf{3}}$ map in the plane confirmed by axis $\boldsymbol{j}_{\mathbf{3}}$ and axis $\boldsymbol{k}_{\mathbf{3}}$. The projection represents the rotation of the link $\mathrm{E}_{i} \mathrm{D}_{i}$ around the axis $\boldsymbol{i}_{\mathbf{3}}$. That is to say, the pitching motions cause space motion of the pitch link, and lead to the rotation between the outer ring and inner ring around the radial direction. $q_{i}$ represents the rotation.

$q_{i}=\arccos \frac{z_{D}}{\sqrt{z_{D}^{2}+y_{D}^{2}}}$

Build a plane with three points $\mathrm{D}_{i}, \mathrm{E}_{i}, \mathrm{~F}_{i}$, and build a coordinate system $\boldsymbol{O}_{\mathbf{5}}-\boldsymbol{i}_{\mathbf{5}} \boldsymbol{j}_{\mathbf{5}} \boldsymbol{k}_{\mathbf{5}}$. The axis $\boldsymbol{i}_{\mathbf{5}}$ is along the link $\mathrm{E}_{i} \mathrm{~F}_{i}$, and axis $\boldsymbol{j}_{\mathbf{5}}$ is perpendicular to link $\mathrm{E}_{i} \mathrm{~F}_{i}$ and located in the plane $\mathrm{D}_{i} \mathrm{E}_{i} \mathrm{~F}_{i}$. In the coordinate system

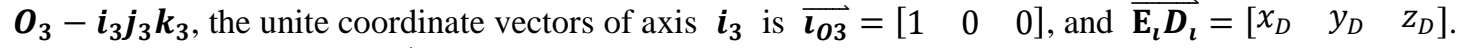

The coordinate of $\overrightarrow{\mathbf{E}_{\boldsymbol{l}} \boldsymbol{D}_{\boldsymbol{\imath}}}$ in the coordinate system $\boldsymbol{O}_{\mathbf{5}}-\boldsymbol{i}_{\mathbf{5}} \boldsymbol{j}_{\mathbf{5}} \boldsymbol{k}_{\mathbf{5}}$ is as follow:

$\overrightarrow{\boldsymbol{E}_{\boldsymbol{\imath}} \boldsymbol{D}_{\mathbf{\imath}}}=\left[\begin{array}{lll}x_{5} & y_{5} & z_{5}\end{array}\right]$

Where $x_{5}=\cos \left\langle\overrightarrow{\boldsymbol{\imath}}, \overrightarrow{\boldsymbol{E}_{\boldsymbol{\imath}} \boldsymbol{D}_{\boldsymbol{\imath}}}\right\rangle \cdot l_{1}=x_{D}, y_{5}=0, z_{5}=\sqrt{l_{1}^{2}-x_{5}^{2}}$.

In the coordinate system $\boldsymbol{O}_{\mathbf{5}}-\boldsymbol{i}_{\mathbf{5}} \boldsymbol{j}_{\mathbf{5}} \boldsymbol{k}_{\mathbf{5}}$, the link $\mathrm{E}_{i} \mathrm{D}_{i}$ is located in the plane confirmed by axis $\boldsymbol{i}_{\mathbf{5}}$ and $\boldsymbol{j}_{\mathbf{5}}$, and use the include angle of link $\mathrm{E}_{\mathrm{i}} \mathrm{D}_{\mathrm{i}}$ and axis $\boldsymbol{i}_{\mathbf{5}}$ to represent the rotation of link $\mathrm{E}_{i} \mathrm{D}_{i}$ around the axis $\boldsymbol{i}_{\mathbf{5}}$. That is to say, the pitching motions cause space motion of the pitch link, and lead to the rotation between the outer ring and inner ring 
around the axial direction. $q_{k}$ represents the rotation:

$$
q_{k}=\arccos \left(\frac{x_{5}}{l_{1}}\right)
$$

\subsection{Motion function of flapping motions}

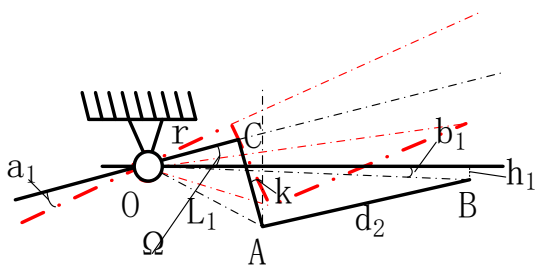

(1)

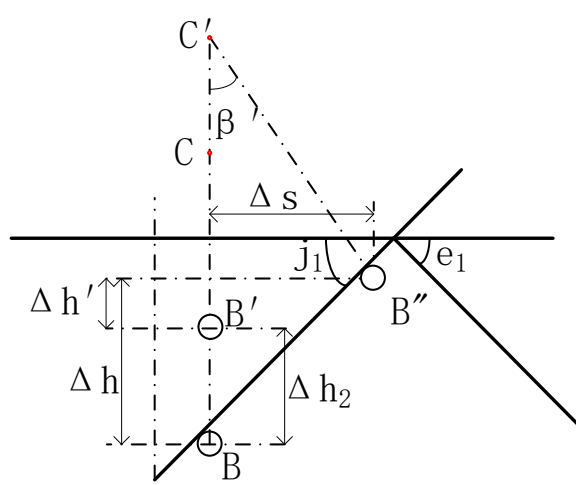

(2)

Figure.7 Movement of flapping mechanism

(1) The projection of first step in plane OCB (2) The right projection of the point B in the second step

The flapping motions are space motions, and passively carried out by the CAM mechanism. Movement of the point B in CAM mechanism is decomposed into two steps. First step, in the initial state, the flapping mechanism rotates an angle $a_{1}$ (that is rotational angle of pitch swing arm) in the plane OAB, and point $\mathrm{B}$ turns to $\mathrm{B}^{\prime}$; second step, the flapping mechanism rotates an angle $\beta^{\prime}$ around the link OC, and point $\mathrm{B}^{\prime}$ arrives to the location $\mathrm{B}^{\prime \prime} . \Delta h$ is the vertical distance from location $\mathrm{B}$ to the position $\mathrm{B}^{\prime \prime}$, and $\Delta h^{\prime}$ is the vertical distance from the position $\mathrm{B}^{\prime \prime}$ to $\mathrm{B}^{\prime}$, and $\Delta \mathrm{S}$ is the horizontal distance from position $\mathrm{B}^{\prime \prime}$ to the plane $\mathrm{BB}$ '. Stipulate $\mathrm{OC}=r, \mathrm{CA}=d_{1}, \mathrm{AB}=d_{2}$, and $\mathrm{OO}^{\prime}$ represents a plane passing though point $\mathrm{O}$, and the underside of plane $0 \mathrm{O}^{\prime}$ is negative, the upper is positive. $\Omega$ represents an included angle between the link OC and the plane $00^{\prime}$ in the initial state. $b_{1}$ represents an included angle between the segment $\mathrm{BO}$ and the plane $0 \mathrm{O}^{\prime}$. According to the geometric relationships, these equations are obtained as follow:

$L_{2}=\sqrt{d_{1}^{2}+\left(r+d_{2}\right)^{2}}$

$b_{1}=\Omega-\arctan \left(\frac{d_{1}}{r+d_{2}}\right)$

In the initial state, the distance from position B to plane $00^{\prime}$ is $h_{1}=L_{2} \sin b_{1}$; the link OC rotates an angle $a_{1}$ around point $\mathrm{O}$, and the distance from point $\mathrm{B}^{\prime}$ to plane $00^{\prime}$ is $h_{2}=L_{2} \sin \left(b_{1}+a_{1}\right)$. So the vertical dimension between location $\mathrm{B}$ and location $\mathrm{B}^{\prime}$ is as follow:

$\Delta h_{2}=h_{2}-h_{1}$

The vertical dimension between location $\mathrm{B}^{\prime \prime}$ and location $\mathrm{B}^{\prime}$ is $\Delta h^{\prime}$.

$\Delta h^{\prime}=d_{1} \cos k-d_{1} \cos \beta^{\prime} \cos k$

Where $k=\Omega+a_{1}$.

The vertical dimension between location B and location $\mathrm{B}^{\prime \prime}$ is as follow:

$\Delta h=\Delta h_{2}+\Delta h^{\prime}=L_{2}\left[\sin \left(b_{1}+a_{1}\right)-\sin b_{1}\right]+d_{1}\left(1-\cos \beta^{\prime}\right) \cos \left(\Omega+a_{1}\right)$

According to the geometrical relationship, the included angle between sliding rail and horizontal plane is represented with $j_{1}$, and $j_{1}=\frac{\pi}{2}-e_{1}$

$\frac{\Delta s}{\Delta h}=\cot j_{1}$

In the plane $\mathrm{B}^{\prime \prime} \mathrm{B}^{\prime} \mathrm{C}^{\prime}$, the point $\mathrm{C}^{\prime}$ is an axis of rotation, and according to the geometrical relationship:

$\frac{\Delta s}{d_{1}}=\sin \beta^{\prime}$ 
Take every formula into the equation (25), the equation (26) is shown as follow.

$L_{2}\left[\sin \left(b_{1}+a_{1}\right)-\sin b_{1}\right]+d_{1}\left(1-\cos \beta^{\prime}\right) \cos \left(\Omega+a_{1}\right)=\frac{d_{1} \sin \beta^{\prime}}{\cot j_{1}}$

Set $A=L_{2}\left[\sin \left(b_{1}+a_{1}\right)-\sin b_{1}\right]+d_{1} \cos \left(\Omega+a_{1}\right), B=d_{1} \tan j_{1}, C=d_{1} \cos \left(\Omega+a_{1}\right)$, the equation (26) is simplified.

$\sqrt{B^{2}+C^{2}} \sin \left(\beta^{\prime}+\omega\right)=A$

Where $\sin \omega=\frac{C}{\sqrt{B^{2}+C^{2}}}, \cos \omega=\frac{B}{\sqrt{B^{2}+C^{2}}}$.

At last, the equation (27) is solved and $\beta^{\prime}$ is obtained in equation (28).

$\beta^{\prime}=\arcsin \left(\frac{A}{\sqrt{B^{2}+C^{2}}}\right)-\arcsin \left(\frac{C}{\sqrt{B^{2}+C^{2}}}\right)$

\subsection{Motion function of testing bearing}

Because the CAM mechanism causes the inner ring of testing bearing rotating, and the direction of rotation of inner ring viewed from the direction of CAM mechanism is opposite with the direction of rotation viewed from the negative of axis $\boldsymbol{i}_{\mathbf{4}}$. The rotation caused by the CAM mechanism is transferred to the coordinate system $\boldsymbol{O}_{\mathbf{3}}-\boldsymbol{i}_{\mathbf{3}} \boldsymbol{j}_{\mathbf{3}} \boldsymbol{k}_{\mathbf{3}}$, so the rotation caused by CAM mechanism is the equation (29).

$\beta^{\prime \prime}=\arcsin \left(\frac{C}{\sqrt{B^{2}+C^{2}}}\right)-\arcsin \left(\frac{A}{\sqrt{B^{2}+C^{2}}}\right)$

The pitching motions cause the rotation between the outer ring and inner ring around the radial direction, and the $\Delta q_{i}$ represents the rotation:

$\Delta q_{i}=\arccos \frac{z_{D}}{\sqrt{z_{D}^{2}+y_{D}^{2}}}-\arccos \frac{z_{D 0}}{\sqrt{z_{D 0}^{2}+y_{D 0}^{2}}}$

Where $z_{D 0}$ and $y_{D O}$ represent the value in the initial state.

So the total rotation around the radial direction caused by the pitching and flapping motions is as follow.

$\eta=\Delta q_{i}+\beta^{\prime \prime}$

$=\arccos \frac{z_{D}}{\sqrt{z_{D}^{2}+y_{D}^{2}}}-\arccos \frac{z_{D 0}}{\sqrt{z_{D 0}^{2}+y_{D 0_{i}}^{2}}}+\arcsin \left(\frac{C}{\sqrt{B^{2}+C^{2}}}\right)-\arcsin \left(\frac{A}{\sqrt{B^{2}+C^{2}}}\right)$

\section{Design of the life testing machine}

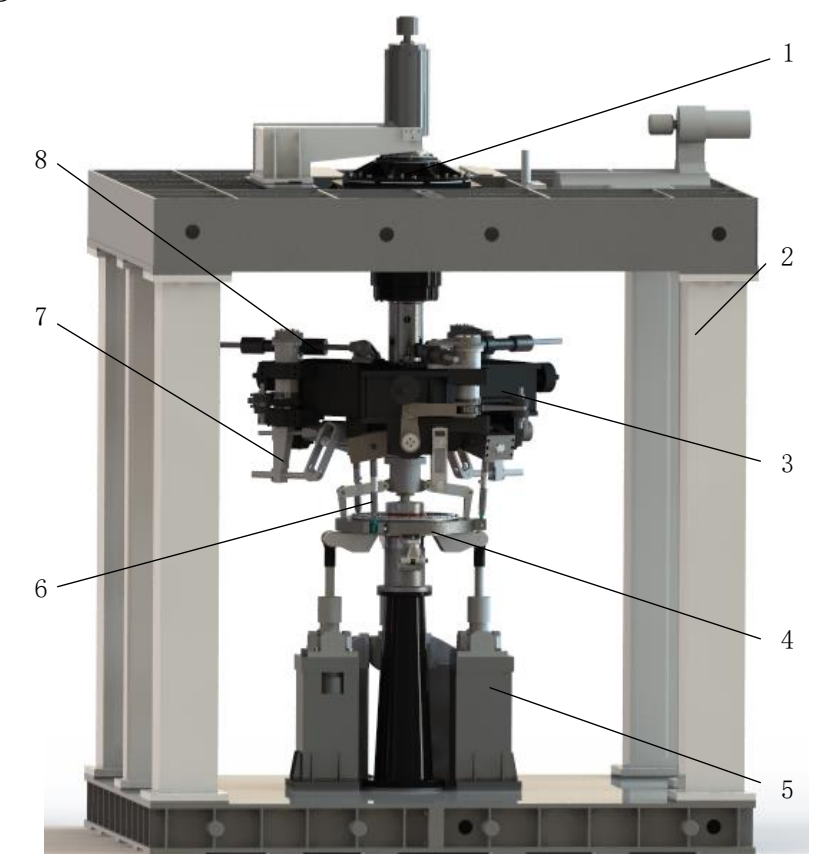

Figure.8 Schematic diagram of life testing machine for self-lubricating rod end spherical plain bearing

1- the main drive system; 2 - framework; 3 - simulation rotating disc; 4 - swash plate system; 5 - boosting system; 6 - pitch link system; 7 - adjusting flapping system; 8 - loading system 
According to the design scheme and the move track analysis, the structure of life testing machine for self-lubricating rod end spherical plain bearing is shown in figure 8 . The life testing machine consists of several systems: the main drive system, framework, simulation rotating disc, swash plate system, boosting system, loading system, adjusting flapping system, pitch link system. Main drive system drives simulation rotating disc to rotate at high speed in order to simulate the rotation of main rotor system; the framework bears all loading and components of life testing machine; the simulation rotating disc is main structure of life testing machine to connect with the main drive system, which can realize high speed rotation around the spindle; the swash plate system includes rotating ring and fixed ring, and the fixed ring connects with the boosting system and adjust the booster to regulate posture of swash plate to implement the total distance and pitching motion. The rotating ring connects with the pitch link system, it cooperates with the simulation rotating disc, and they rotate together around the spindle; Boosting system is composed by parallel mechanism and used to adjust the position of swash plate; Loading system is consisted by hydraulic device to simulate the resistance in the process of helicopter's flight.

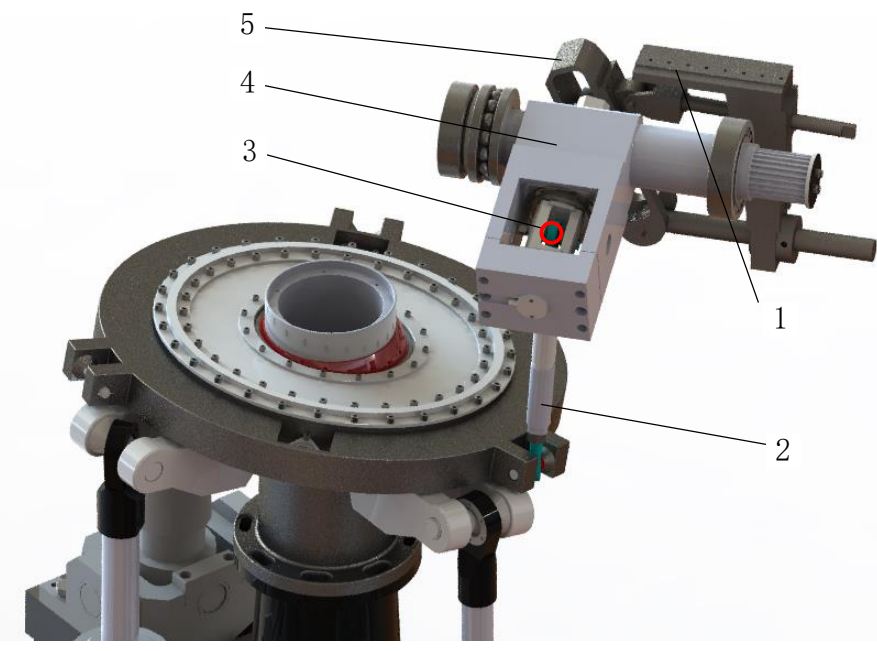

Figure.9 Structure of branched chain

1 -adjusting flapping mechanism; 2 -pitch link; 3 - testing bearing; 4 -pitch swing arm; 5 -the chute of adjusting flapping system

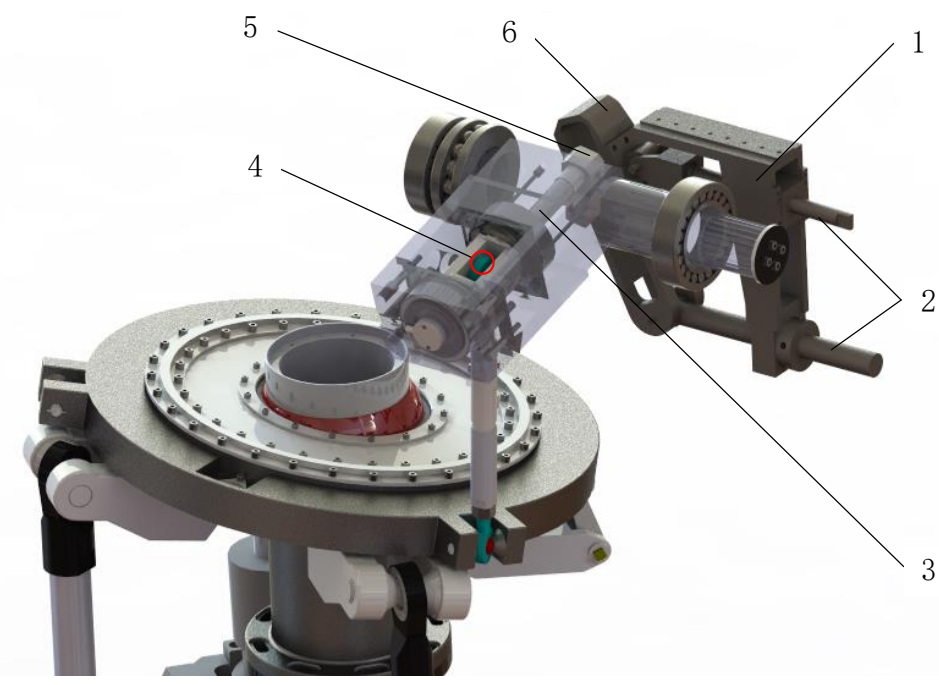

Figure.10 Schematic diagram of adjusting flapping mechanism

1 - the substrate of adjusting flapping; 2 - the adjustment screws of adjusting flapping mechanism; 3 - pitch swing arm spindle; 4 - testing bearing; 5 - adjusting flapping arm; 6 - the chute of adjusting flapping

Pitch link system has three support arms, they distribute equally on a circle. The pitch link system includes pitch link and pitch swing arm. The testing bearing is installed on intersection of pitch link and pitch swing arm spindle, and it connects with the pitch link and adjusting flapping system. Adjusting flapping system is shown in the figure 10. The substrate of adjusting flapping is installed on the bottom of the simulation rotating disc, and adjust the adjustment screws of adjusting flapping mechanism, it can adjust the location and tilt angle of the chute of adjusting flapping to 
realize the flapping motions. The adjusting flapping arm is installed on the end of pitch swing arm spindle, and a track roller is fixed on the adjusting flapping arm. The track roller is tangent with the chute of adjusting flapping. When the pitch swing arm swings, it leads to the track roller to move along the chute, and it will drive the adjusting flapping arm and pitch swing arm spindle to rotate, and realize the flapping motions. The principle of the process is shown in figure 11. In the figure (a), the end of pitch link is located in high state and pitch swing arm swings upward, and track roller fixed on the adjusting flapping arm moves along the chute, at the same time the adjusting flapping arm is located in the left side of the vertical direction, and the inner ring of testing bearing drove by pitch swing arm spindle rotates in figure (b). The rotating ring of swash plate rotates counterclockwise to the figure (c), the end of pitch link is located in the low, and pitch swing arm swings downward, it drives the adjusting flapping arm, and the adjusting flapping arm is located in the right side of the vertical direction, the inner ring of testing bearing drove by pitch swing arm spindle rotates in figure (d).

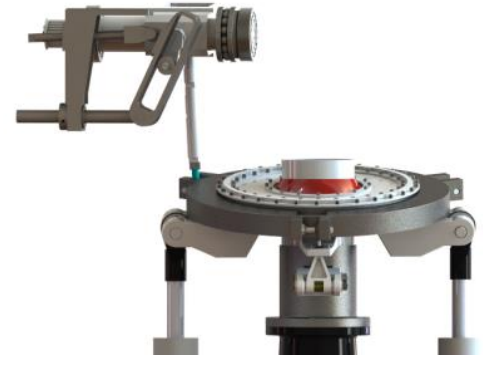

(a)

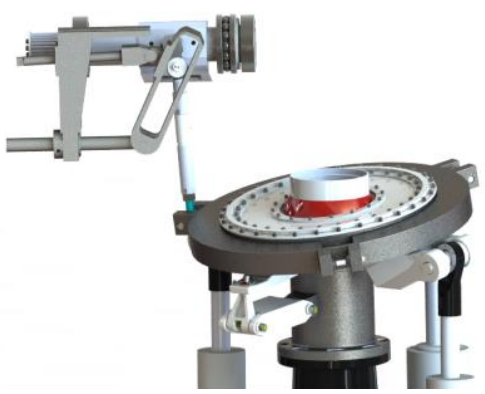

(c)

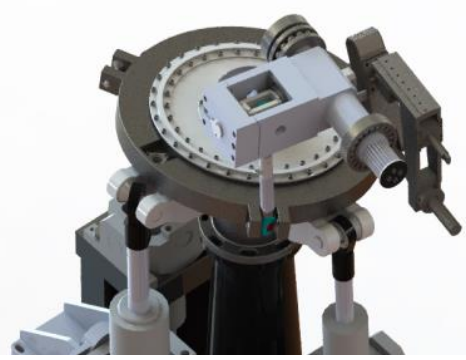

(b)

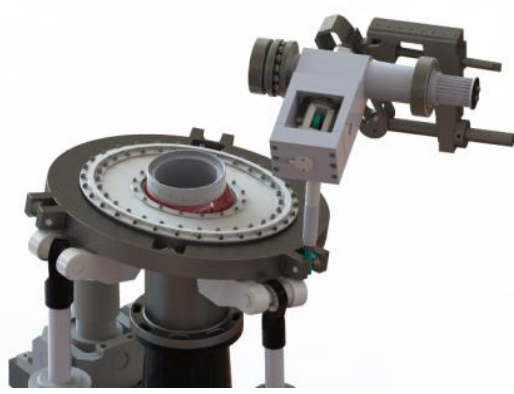

(d)

Figure.11 The working principle diagram of adjusting flapping mechanism

\section{Lifetime measurement of self-lubricating rod end spherical plain bearing}

When it applies a testing, it needs to determine whether self-lubricating fabric liner wears out and loses efficacy. Measuring the friction coefficient of testing bearing, measuring temperature of testing bearing and measuring the wear extent are used to evaluate friction performance of testing bearing in experiment (Liu, et al., 2012 ).

In the design scheme, measuring temperature of testing bearing is used to evaluate lifetime of bearing, and the temperature sensor is used to measure temperature. Because the self-lubricating rod end spherical plain bearing needs to bear multidimensional loading, it can't determine the exact location of the contact between inner ring and outer ring of spherical plain bearing. So the temperature sensor is placed in rotation centerline of pin which is assembled with inner ring. A deep hole is designed in rotation centerline of pin, and the deep hole is enough deep to make sure the sensor can reach the whole testing bearing. Then temperature sensor is installed in the deep hole as shown in the Figure 12 , and it can measure the temperature of testing bearing in real time.

The relative movement is occurred between inner ring and outer ring of spherical plain bearing, and friction generates heat. It is the cause for temperature rising, and it means the testing bearing is in the run-in period. When the temperature gently rises, the heat reaches a balance, and the testing bearing is in the stable period. When the temperature suddenly sharp increases, it means the liner of testing bearing has worn through, and the testing bearing is failure. 


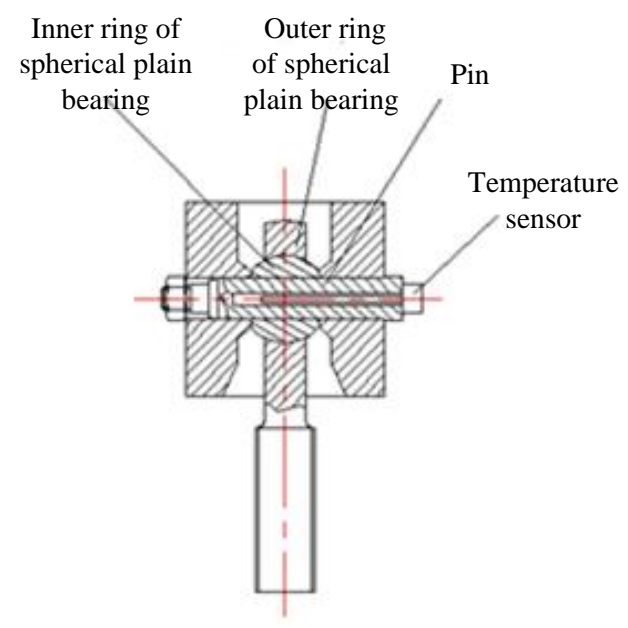

\section{Simulation of motion function}

Figure.12 Lifetime measurement of self-lubricating rod end spherical plain bearing

According to the formula derivation, and the input parameters are set as $Z_{c}=30, \psi=30^{\circ}, \varphi=5^{\circ}$, use MATLAB to carry out the simulation calculation, the curve of motion function about the outer ring of testing bearing rotating around the axial and radial direction relative to the inner ring is obtained, when self-lubricating rod end spherical plain bearing sustains the pitching and flapping motions.

Because the structure of three support arms is consistent, the model is simplified appropriately, and preserve the moving parts of one support arm. According to setting input variable, we carry out the motion simulation. When swash plate revolves a lap, measure the value of outer ring of testing bearing rotating around the axial and radial direction relative to the inner ring, then process data and compare with curve of motion function.

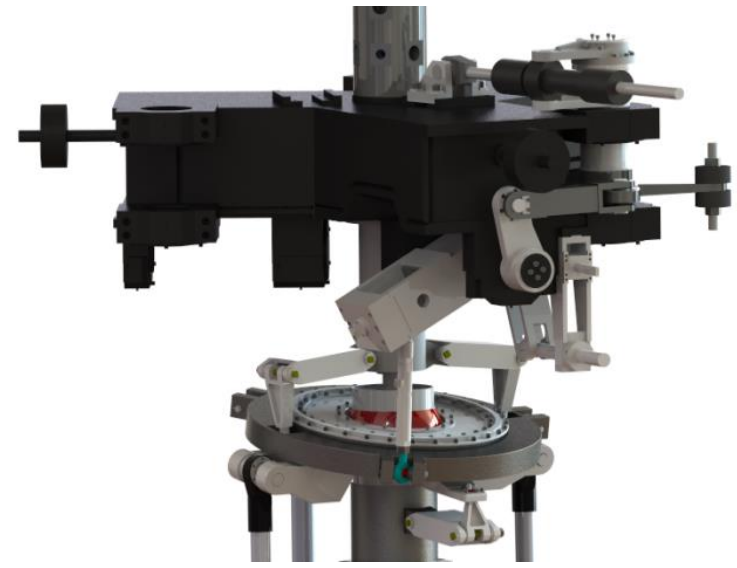

Figure.13 Motion simulation model of the life testing machine

The curve of motion function about the outer ring of testing bearing sustaining the pitching motions rotating around the axial direction relative to the inner ring and the data of the motion simulation are shown in the figure 14 . Comparing the difference value of peaks and troughs of the curve $b$ with the difference value of peaks and troughs of curve $\mathrm{d}$, it gives a difference of just $1.1 \%$, and it shows that use the measured data about the rotational angle of pitch swing arm to replace the measured data about the variation of the outer ring of testing bearing sustaining the pitching motions rotating around the axial direction relative to the inner ring. It simplified the measuring mechanism to realize online measurement.

Figure 15 shows the change frequency that the outer ring of testing bearing rotates around the radial direction relative to the inner ring is twice the rotational frequency of the swash plate. The change frequency of testing bearing caused by the flapping motions coincides with the rotational frequency of the swash plate. Contrast the motion simulation measurement data and the curve of function, it certifies that they have good degree of fitting, which proves the accuracy of motion function. 


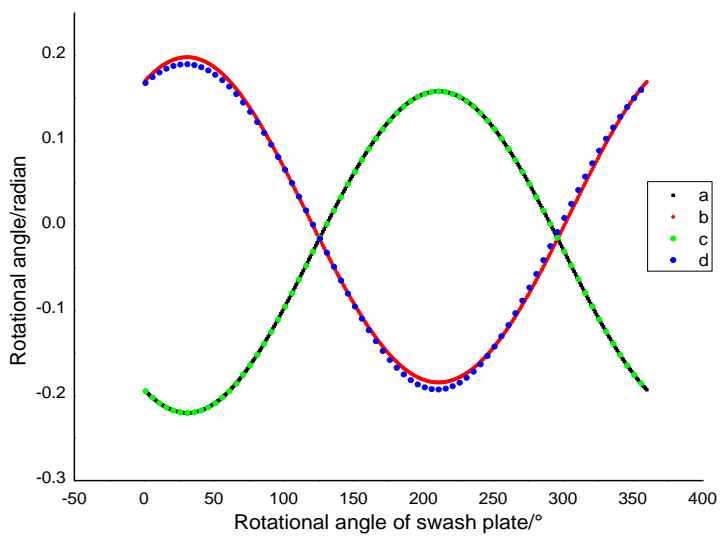

Figure.14 The comparison diagram between the curve of function and motion simulation data about the rotational angle of pitch swing arm and the testing bearing rotating around the axial direction

a-the curve of function about rotational angle of pitch swing $\operatorname{arm} \mathrm{E}_{\mathrm{i}} \mathrm{F}_{i}$; b-the curve of function about the outer ring of testing bearing rotate around the axial direction relative to the inner ring; c-the motion simulation data of rotational angle of pitch swing arm $\mathrm{E}_{\mathrm{i}} \mathrm{F}_{i}$; d-the motion simulation data of the outer ring of testing bearing rotate around the axial direction relative to the inner ring.

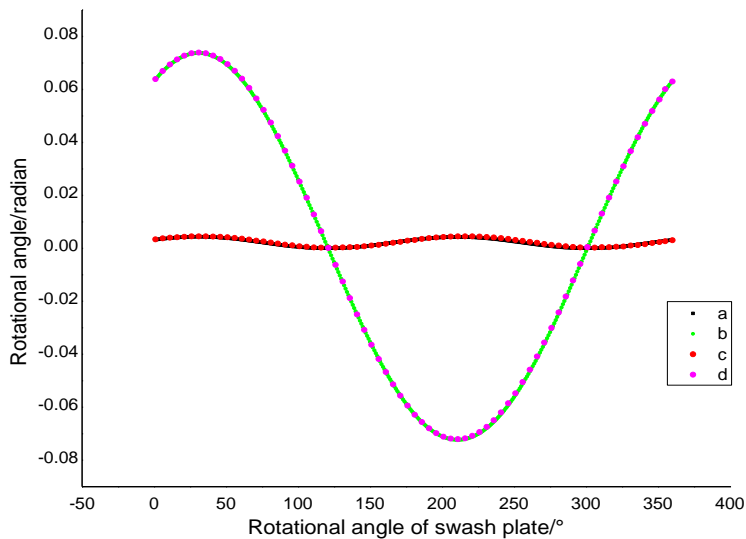

Figure.15 The comparison diagram between curve of function and motion simulation data of testing bearing rotating around the radial direction

a-the curve of function about the testing bearing rotating around the radial direction caused by the pitching motions; b-the curve of function about the testing bearing rotating around the radial direction caused by the flapping motions; c-the motion simulation of the testing bearing rotating around the radial direction caused by the pitching motions; d-the motion simulation

\section{Conclusion} of the testing bearing rotating around the radial direction caused by the flapping motions.

(1) In this paper, we put forward a novel design about the life testing machine for self-lubricating rod end spherical plain bearing and give a method to test the lifetime of testing bearing, and analyze the DOF and the forward displacement of mechanism.

(2) In the paper, we analyze the movement trail of self-lubricating rod end spherical plain bearing when the life testing machine is working, and obtain the function about the outer ring of testing bearing sustaining the pitching and flapping motions rotating around the axial and radial directions relative to the inner ring. It provides a theory basis for accurately applying the loading on the testing bearing and boundary conditions for optimizing the mechanism structure.

(3) According to the mechanism design, we complete the new life testing machine for self-lubricating rod end spherical plain bearing of the helicopter and finish the motion simulation of life testing machine, and it proves that the life testing machine implements to simulate the pitching and flapping motions of self-lubricating rod end spherical plain bearing on the ground.

\section{References}

Fan Xiaowang, He Liangjia, Pao Fei and so on. Alternating load spherical plain bearing life testing machine: China, 201120195643.8[P]. (2011-06-13). 
GaoYadong, Zhang Zengchang, Yu Jianhang. Diagnosis of worn pitch-change-link rod end in helicopter rotor system. [J]. Journal of Nan jing University of Aeronautics \& Astronautics, VOL.38, NO.1(2006), pp.6-10(in Chinese).

Guo Xijuan, Huang Tianyu, Chang Fuqing. Kinematics of a new hybrid mechanism with reduced DOF. and nested dynamic connection. [J]. Journal of mechanical engineering, VOL.49, NO.1 ( 2013), pp.1-7(in Chinese).

Jonathan A. Keller, Paul Grabill. Inserted fault vibration monitoring tests for a CH-47D aft swashplate bearing.[J]. the American Helicopter Society 61st Annual Forum. (2005).

Lancaster J K. The Influence of Reversing Loads on the Performance of Self-Lubricating, Dry Bearings[R]. UK, Royal Aircraft Establishment, (1983),pp.296 -307.

Lin Hao, Chen Hui. Development need and status of variable speed transmission for helicopter[J]. Aeronautical Manufacturing Technology. NO,8(2016),pp.46-47.

Liu Jian, Zhang Yongzhen, Du Sanming and so on. Temperature and friction characteristics of PTFE braided composites[J]. Journal of Mechanical Engineering, VOL.48, NO.23(2012),pp.90-94.

McFalls M, White D, Army U S. Lead the fleet implementation of structural usage monitoring [C] / /Proceeding s of the American Helicopter Society 60th Annual Forum. Baltimore, M D: The America n Helicopter Society, (2004a).

McFalls M, White D, Jackson A. Fatigue life analysis of helicopter components based on usage monitoring .[C]. // Proceedings of the American Helicopter Society 60th Annual Forum. Baltimore, M D: The American Helicopter Society, (2004b).

Qin Chao,Wang Xinmin,Zhao Kairui. Design of rotor speed regulator system based MCU C8051F005.[J]. Measurement \& Control Technology, NO.9(2007), pp.29-70(in Chinese).

Shimizu T, Katsuma H, Ito S, et al. Self-Lubricating Spherical Plain Bearing for Heavy Duty Application[R] . Toyo, NTN Toyo Bearing Co., Ltd., (1982).

Wang Xuelei, Liu Feng, Du Xiong, Guo Xinjuan. Analysis of freedoms of helicopter swash plate's equivalent parallel mechanisms. [J]. Machinery design \& manufacture, No.1(2014), pp.59-61(in Chinese).

Wei Libao, Huang Shijun, Yang Yulin. The development of spherical plain bearing tester for swash plate.[J]. Helicopter technique, NO.2(2008), pp.35-38(in Chinese).

Xijuan Guo, Dayu Zhang, Qingjia Geng, et al. The synthesis of dynamic junction over-constrained parallel-parallel mechanism based on the weighted directed graph theory.[J]. ICIC Express Letters, VOL.7, NO.11,(2013), pp.3057-3065.

Zhou Kai. Spherical plain bearing life testing machine design and precision compensation for testing wear. [D]. China Qin huangdao: Yanshan university,(2012).

Zhou Yuan, Guo Xijuan. 3-SPS+RRS+PS mechanism analysis and hessian matrix solution. [J].International Conference on Logistics Engineering, Management and Computer Science(LEMCS 2014), pp.1030-1036. 